\title{
PGE1 triggers Nrf2/HO-1 signal pathway to resist hemin-induced toxicity in mouse cortical neurons
}

\author{
Jiabing Shen ${ }^{1,2 \#}$, Mao-Sheng Cao ${ }^{1,2 \#}$, Tingting Zhou ${ }^{1,2}$, Ying Chen ${ }^{1,2}$, Jingjing Liang ${ }^{1,2}$, Yan Song ${ }^{3}$, \\ Chengbin Xue ${ }^{2,4}$, Mao-Hong $\mathrm{Cao}^{1}$, Kaifu $\mathrm{Ke}^{1}$ \\ ${ }^{1}$ Department of Neurology, Affiliated Hospital of Nantong University, Nantong, China; ${ }^{2}$ Jiangsu Clinical Medicine Center of Tissue Engineering \\ and Nerve Injury Repair, Research Center of Clinical Medicine, Affiliated Hospital of Nantong University, Nantong, China; ${ }^{3}$ Department of \\ Neurology, Nantong Hospital of Traditional Chinese Medicine, Nantong, China; ${ }^{4}$ Key Laboratory of Neuroregeneration, Ministry of Education and \\ Jiangsu Province, Co-innovation Center of Neuroregeneration, Nantong University, Nantong, China \\ Contributions: (I) Conception and design: K Ke, MH Cao, C Xue; (II) Administrative support: None; (III) Provision of study materials or patients: J \\ Shen, MS Cao; (IV) Collection and assembly of data: J Shen, MS Cao, C Xue; (V) Data analysis and interpretation: Y Chen, J Liang, Y Song; (VI) \\ Manuscript writing: All authors; (VII) Final approval of manuscript: All authors. \\ \#These authors contributed equally to this work. \\ Correspondence to: Kaifu Ke; Maohong Cao; Chengbin Xue. Department of Neurology, Research Center of Clinical Medicine, Affiliated Hospital of \\ Nantong University, 20 Xisi Road, Nantong, China. Email: kekaifu_nt@126.com; cmhongnt@sina.com; xuechengbin@ntu.edu.cn.
}

Background: Prostaglandin E1 (PGE1) exerts various pharmacological effects such as membrane stabilization, anti-inflammatory functions, vasodilation, and platelet aggregation inhibition. We have previously demonstrated that PGE1 has a beneficial impact on patients suffering from intracerebral hemorrhage (ICH). The related mechanism underlying PGE1's beneficial effect on ICH treatment needs further exploration.

Methods: The present study elucidates the mechanism of PGE1 on ICH treatment using a neuronal apoptosis model in vitro. The mouse primary cortical neurons were pretreated with different concentrations of PGE1, followed by the treatment with hemin, the main catabolite in whole blood, to mimic the clinical ICH.

Results: Comparing with the vehicle-treated group, PGE1 prevented cultured cortical neurons from the accumulation of inhibited intracellular levels of reactive oxygen species (ROS), amelioration of mitochondrial membrane potential, and hemin-induced apoptosis. The reduction of ROS and apoptosis were associated with the up-regulation of Heme oxygenase-1 (HO-1) expression. Knockdown of nuclear transcription factor erythroid 2-related factor (Nrf2) by siRNA attenuated the upregulation of HO-1 as well as the protective effect of PGE1.

Conclusions: Our work suggests that the Nrf2/HO-1 molecular pathway may play a crucial role in treating ICH patients with PGE1 and may represent novel molecular targets, resulting in discovering new drugs for ICH treatment.

Keywords: Apoptosis; Nrf2; PGE1; intracerebral hemorrhage (ICH); oxidative stress

Submitted Aug 12, 2020. Accepted for publication Jan 29, 2021.

doi: $10.21037 / \mathrm{atm}-20-5839$

View this article at: http://dx.doi.org/10.21037/atm-20-5839

\footnotetext{
^ ORCID: 0000-0003-1378-672X.
} 


\section{Introduction}

Although medical and surgical procedures are in place to follow, intracerebral hemorrhage (ICH) remains the most deadly and least treatable stroke type $(1,2)$. The fatality ratio is approximately $40 \%$ during the first month of $\mathrm{ICH}$, with no improvement in the past 20 years (3). Therefore, exploring the pathogenesis of post-ICH-induced brain injury may ameliorate ICH's clinical prognosis $(4,5)$. The vascular rupture in the brain parenchyma leads to primary and secondary damage following ICH (4). The cytotoxic, oxidative stress, excitotoxic, inflammatory pathways all contribute to the tissue damages that may eventually lead to disability or even death after ICH (6).

As a biologically active lipid mediator produced by pericytes, smooth muscle, endothelium, mast cells, fibroblasts, platelets, and leucocytes, prostaglandin E1 (PGE1) has multiple biological effects such as membrane stabilization, anti-inflammatory functions, vasodilation, and inhibition of platelet aggregation $(7,8)$. For the therapy of patients with peripheral arterial occlusive disease, PGE1 has played broad roles in clinical practices $(9,10)$. Clinical and pre-clinical evidence showed the benefits of PGE1 on curing ischemic heart disease and cerebral ischemia (8,11-13). We have previously tried to use PGE1 to treat ICH patients in the clinic and have achieved positive protective results (14).

The exact mechanism underlying PGE1's beneficial effect on ICH remains elusive. PGE1 may increase c-Fos and Myc proteins' expression and protect rat hippocampal cells from hypoxic injury $(15,16)$, and prevent neuronal apoptosis in the rat spinal cord induced by sciatic nerve constriction $(17,18)$. PGE1 may also effectively protect cortical neurons from glutamate cytotoxicity (19).

To further investigate the molecular mechanism underlying PGE1's beneficial effect on ICH, here we have explored the molecular mechanism of PGE1 in neuronal protection using an ICH model in vitro. Mouse primary cortical neurons were treated with hemin to establish the clinical ICH pathological model. As a hemoglobin product, hemin can induce ROS by releasing the ferrous iron intracellularly (20). Heme oxygenase 1 (HO-1), a stress-inducible enzyme, accelerates hemin's degradation to produce strong antioxidant bilirubin (21-23). HO-1 vigorously protects neurons against heme-mediated damage in vitro and exerts beneficial effects on heme clearance in vivo $(24,25)$. HO-1 levels are deficient under physiological conditions, which can be up-regulated by oxidative stress via Nrf2-regulated transcriptional activity in the CNS (26). This work comprehensively evaluated the protective efficacy of PGE1 in vitro and explored its molecular mechanism via investigating cell viability, apoptosis, and $\mathrm{HO}-1 / \mathrm{Nrf} 2$ expression.

We present the following article in accordance with the ARRIVE reporting checklist (available at http://dx.doi. org/10.21037/atm-20-5839).

\section{Methods}

\section{Drugs and reagents}

Dulbecco's modified eagle's medium (DMEM) and Fetal bovine serum (FBS) were purchased from Gibco (Grand Island, NY). PGE1, hemin, Hoechst 33342, 2'7'dichlorofluorescein diacetate (DCFH-DA), trypsin, and In Situ Cell Death Detection Kit was purchased from Sigma (St. Louis, MO). The antibodies of Anti-NeuN, antiHO-1, anti-Nrf2, anti-Cleaved caspase-3, anti-Bcl-2, antiBax, anti-Cyt C, anti- $\beta$-actin, and TMRE-Mitochondrial Membrane Potential Assay Kit were purchased from Abcam (Cambridge, MA). HRP-conjugated goat anti-mouse IgG or donkey anti-rabbit IgG were obtained from Cell Signaling Technology (Danvers, MA). The kits of Reactive Oxygen Species Assay and Caspase-3/CPP32 Colorimetric Assay were provided by Beyotime (Nantong, China). Cell Counting Kit-8 (CCK-8) was purchased from Dojindo (Kumamoto, Japan).

\section{Cell treatment and siRNA transfection}

All animal experiments in this study were approved by the Administration Committee of Experimental Animals [SYXK (Su) 2012-0031] of Jiangsu in compliance with the National Institutes of Health Guidelines for the Care and Use of Laboratory Animals. Following previous protocol, primary cortical neurons were dissociated from C57BL/6 mouse cortex at embryonic day $15(27,28)$. In brief, the cortical tissue was dissected on an ice-board and digested in $0.25 \%$ trypsin in hank's balanced solution free of $\mathrm{Ca}^{2+}$ and $\mathrm{Mg}^{2+}$ at $37^{\circ} \mathrm{C}$ for $7 \mathrm{~min}$. The upper suspension was then filtered and centrifuged with a centrifugal machine at $800 \mathrm{rpm}$ for $5 \mathrm{~min}$. The sediment was further resuspended in DMEM with a $10 \%$ FBS supplement. The suspension was then cultured at a density of $8 \times 10^{5}$ cells/well in a 96 -well plate or a density of $2 \times 10^{4}$ cells/well in a 6 -well plate. After plating, the medium was replaced with NEUROBASAL ${ }^{\mathrm{TM}}$, which 
contained $0.5 \mathrm{mM}$ glutamine, $2 \% \mathrm{~B} 27,1 \%$ antibioticantimycotic, and changes every three days for neuron maturation.

To simulate ICH injury in vitro, the cells were exposed to hemin at the different final concentration $(1,10,50$, and $100 \mu \mathrm{M})$ and incubated for $24 \mathrm{~h}$. Cell viability and apoptosis were analyzed. Neurons were pretreated with PGE1 at $10 \mathrm{nM}, 100 \mathrm{nM}, 1 \mu \mathrm{M}$, and $10 \mu \mathrm{M}$ for $12 \mathrm{~h}$, followed by exposure to hemin at an optimal concentration for another $24 \mathrm{~h}$. The cells with no treatment were appointed as control.

According to the manufacturer's instructions (Invitrogen, USA), primary cortical neurons were transfected with small interfering RNA (si-Nrf2) specific to Nrf2 (5'-GACATAGATCTTGGAGTAAGT-3', GenePharma, China) using Lipofectamine ${ }^{\circledR} 2,000$ transfection reagent. Western blotting was applied to evaluate Nrf2 interfering efficiency. Forty-eight hours before hemin stimulation, the neurons were transfected with si-NC (negative control siRNA) or si-Nrf2 $(50 \mathrm{nM})$. Treated cells were harvested and analyzed with western blotting and intracellular ROS accumulation.

\section{Assay of cell viability}

The viability of neurons was examined using CCK-8, as recommended by the manufacturer. Briefly, cells were cultured onto a 96 -well plate at a density of $2 \times 10^{4}$. The cells were pretreated with PGE1 for $12 \mathrm{~h}$, followed by hemin administration for another $24 \mathrm{~h}$. Then CCK-8 solution was added to each well at a final concentration of $10 \%$ (v/v). The cells' absorbance was measured at 490/630 nm by a microplate reader (Bio-Rad) after an additional $2.5 \mathrm{~h}$ incubation at $37^{\circ} \mathrm{C}$.

\section{Cytotoxicity assessment}

Cytotoxicity was determined by measuring extracellular lactate dehydrogenase (LDH) activity according to the manufacturer's specifications. Briefly, primary cortical neurons were seeded onto a 6-well plate pretreated with or without PGE1 before hemin exposure. With a microplate reader (Bio-Rad), the culture medium's LDH activity was tested by measuring absorbance at $490 \mathrm{~nm}$.

\section{Detection of mitochondrial membrane potential (MMP) and ROS production}

The MMP was detected by an indirect approach. Briefly, the fluorescent dye TMRE at the concentration of $20 \mathrm{nM}$ was added to the cultured neurons for $20 \mathrm{~min}$ at $37^{\circ} \mathrm{C}$. The cells were then washed with PBS solution twice, followed by image acquisition under a fluorescent microplate with excitation/emission wavelength at 530/590 nm. The relative fluorescence intensity was analyzed using a microplate reader (ELx-800).

The DCFH-DA-based assay was used to evaluate the intracellular ROS level. DCFH-DA at the concentration of $20 \mu \mathrm{M}$ was added to the 6-well plate and incubated at $37^{\circ} \mathrm{C}$ for $30 \mathrm{~min}$ in the dark. After a gentle rinse for three times with PBS, the fluorescence of dichlorofluorescein (DCF), an oxidation product of DCFH-DA, and the reflection of the intracellular ROS level, was measured on a microplate reader with excitation/emission wavelength at $485 / 528 \mathrm{~nm}$ (ELx-800).

\section{Assay of apoptosis}

The primary cortical neurons were seeded onto glass coverslips at a density of $5 \times 10^{4}$ cells/well. After fixation for 20 min by paraformaldehyde (4\%) at room temperature, terminal deoxynucleotidyl transferase dUTP nick end labeling (TUNEL) and Hoechst 33,342 staining were performed. Stained cells were observed under a DMR fluorescence microscope with excitation/emission wavelength at $340 / 510 \mathrm{~nm}$. The ratio of fragmented nuclei to total cells was defined as the apoptotic ratio.

\section{Assay of caspase-3 activity}

According to the manufactory's instructions, the caspase- 3 activity was determined by the caspase-3/CPP32 colorimetric assay kit. Cultured neurons at different groups were homogenized and quantified by BCA analysis. The supernatant protein $(120 \mu \mathrm{g})$ was mixed with an equal volume of $2 \times$ reaction buffer and $200 \mu \mathrm{M}$ DEVD-pNA substrate. The absorbance was measured by ELx-800 microplate reader at $405 \mathrm{~nm}$ after $1-2 \mathrm{~h}$ reaction at $37^{\circ} \mathrm{C}$.

\section{Western blot analysis}

Primary cortical neurons were lysed in a modified RIPA lysis buffer (including protease inhibitor, phosphatases inhibitor, $5 \mathrm{mM}$ EDTA, $1 \%$ sodium deoxycholate, $1 \%$ sodium dodecyl sulfate (SDS), $50 \mathrm{mM}$ Tris, $\mathrm{pH} \mathrm{7.5,1 \%}$ $\mathrm{NP}-40$, and $150 \mathrm{mM} \mathrm{NaCl})$. Cell extracts were clarified by centrifugation at $12,000 \times \mathrm{g}$ for $15 \mathrm{~min}$ to collect the 
supernatant. Following detecting concentration with the BCA assay, total protein samples (30 $\mu \mathrm{g}$ each) from cell lysates were subjected to $10 \%$ SDS-polyacrylamide gel electrophoresis (SDS-PAGE) and transferred to a polyvinylidene difluoride (PVDF) filter membrane. Blocked by $5 \%$ non-fat milk, the membrane incubated with antiBax antibody (1:1,000, rabbit), anti-Bcl-2 antibody (1:500, mouse), anti-cleaved caspase- 3 antibody (1:1,000, rabbit), anti-Cyt C antibody (1:500, rabbit), anti-HO-1 antibody (1:1,000, rabbit), anti-Nrf2 antibody (1:2,000, rabbit) and anti- $\beta$-actin $(1: 1,000$, mouse) overnight. After washing with TRIS-buffered saline/Tween $(0.1 \%$ Tween 20 , $150 \mathrm{mM} \mathrm{NaCl}, 10 \mathrm{mM}$ TRIS, $\mathrm{pH}$ 8.0), the membranes were incubated with HRP-conjugated donkey anti-rabbit IgG antibodies or HRP-conjugated goat anti-mouse IgG for one hour and visualized with enhanced chemiluminescence (ECL) kit (Pierce).

\section{Statistical analysis}

The data were analyzed by IBM SPSS Statistics 26.0 (IBM Corp., Armonk, NY, USA) and expressed as mean \pm standard error of the mean (SEM). The statistical analysis was implemented with One-way ANOVA as well as Tukey's multiple comparison tests. $\mathrm{P}<0.05$ was considered significant.

\section{Results}

\section{Neurotoxicity induced by Hemin in primary cortical neurons}

Cell morphological observation and CCK-8 assay may evaluate cell viability to investigate the neurotoxicity induced by hemin on primary cortical neurons. Hemin dose-dependently decreased cell viability (Figure $1 A$ ). At the concentration of $50 \mu \mathrm{M}$, hemin appeared to reduce the cell viability in a time-dependent manner (Figure 1B). Under a phase-contrast microscope, cells that have not been treated or DMSO (control) exhibited a normal neuronal shape with oval cell bodies, complete cell membranes, and neural networks. The hemin-treated cells exhibited early apoptotic morphology, such as vacuoles emergence, cell shrinkage, and disappearance of neurite protrusions (Figure 1C). Based on the previous work (29), $50 \mu \mathrm{M}$ of hemin appeared to be optimal in inducing apoptosis and was thus selected for the subsequent experiments in our work.

\section{PGE1 pretreatment confers resistance to hemin-induced damage in primary cortical neurons}

To explore whether PGE1 gain protecting effect on injured neurons, primary cortical neurons were pretreated with $10 \mathrm{nM}, 100 \mathrm{nM}, 1 \mu \mathrm{M}, 10 \mu \mathrm{m}$, or without PGE1 for $12 \mathrm{~h}$. Then they were exposed to $50 \mu \mathrm{M}$ hemin for $24 \mathrm{~h}$, and the cell viability was examed by CCK-8, LDH assays, and morphological observation. PGE1 pretreatment at 10 or $100 \mathrm{nM}$ prevented neurons from hemin-induced toxicity (Figure 2A). Moreover, LDH releasing assay (Figure 2B) indicated that PGE1 at the concentration of 10 or $100 \mathrm{nM}$ significantly reduced LDH releasing and cell death $(\mathrm{P}<0.05)$. Salvaged neurons showed healthy, unbroken cell body and connections to each other in neural networks (Figure 2C).

\section{PGE1 ameliorated mitochondrial function and reduced ROS production in bemin-injured cortical neurons}

The decreased MMP is an essential indicator for apoptosis due to its critical role in maintaining the physiological function of the respiratory chain supercomplex (30). TMRE fluorescence was measured to investigate the changes of MMP in hemin-injured cortical neurons. The results revealed that the toxicity of hemin reduced TMRE fluorescence intensity by $47 \%$ compared with control group, indicating mitochondrial depolarization (Figure 3A). Pretreatment with 10, $100 \mathrm{nM}$ of PGE1 alleviated this depolarization (Figure 3B). Interestingly, higher concentrations of PGE1 $(1$, or $10 \mu \mathrm{M})$ were not efficacious (Figure 3B). In the DCFH-DA assay, PGE1 at 10 , or $100 \mathrm{nM}$ significantly decreased hemin-induced intracellular ROS accumulation (Figure 3C). All these results suggested that PGE1 pretreatment significantly reduced the ROS production in hemin-injured cortical neurons.

\section{PGE1 rescued primary cortical neurons from bemin- induced apoptosis}

To explore the potential mechanisms underlying the protective effect of the PGE1on hemin-induced apoptosis model of primary cortical neurons, we further investigate the expression of caspase-3, Bcl-2/Bax, and Cyt $\mathrm{C}$ by western blotting analysis. Pretreatment of primary cortical neurons with 10 or $100 \mathrm{nM}$ PGE1 for $12 \mathrm{~h}$ significantly decreased hemin-induced levels of cleaved caspase- 3 , an 

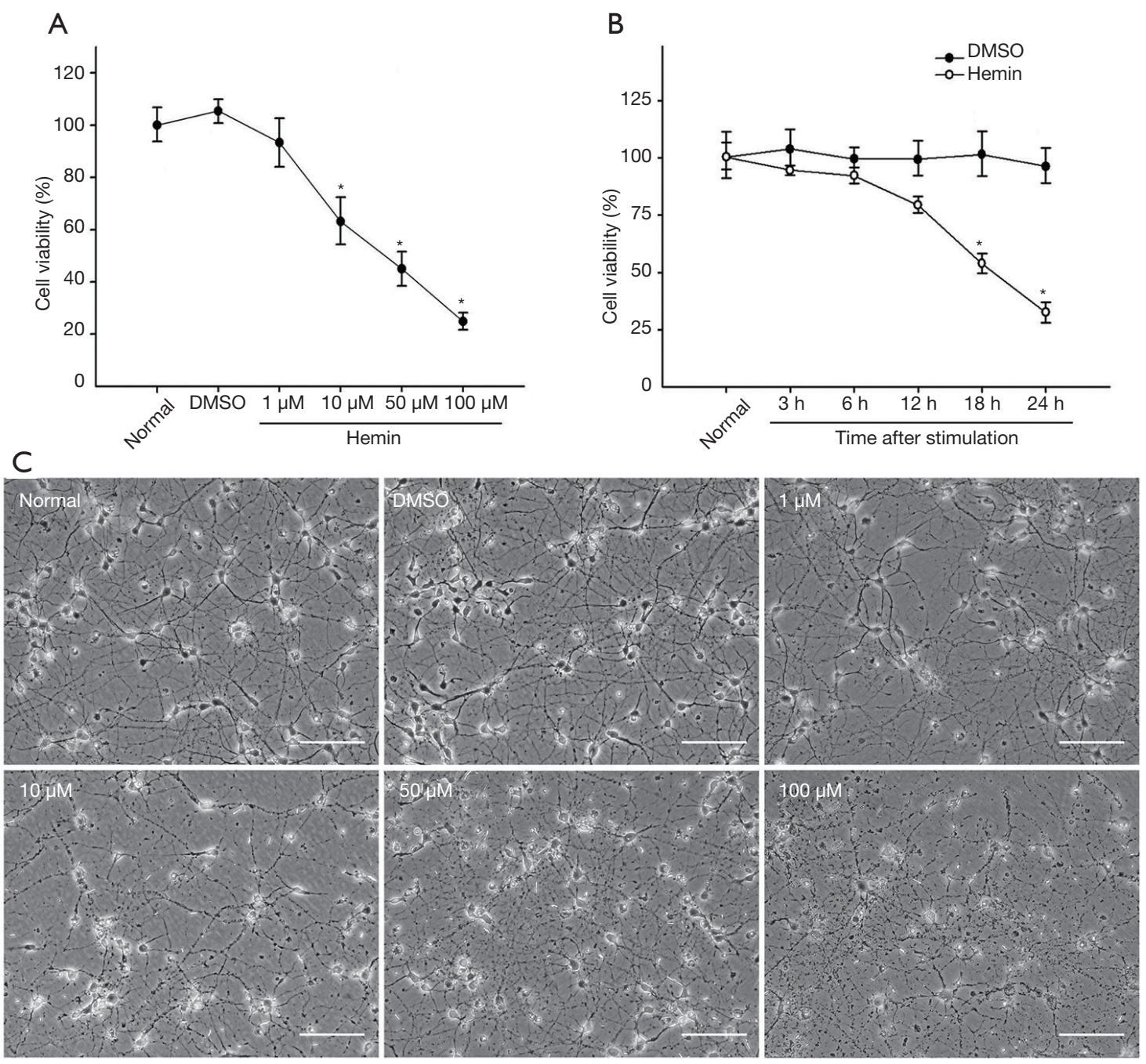

Figure $1 \mathrm{Hemin}$ induced injury in primary cortical neurons. (A) After exposure to different hemin concentrations for $24 \mathrm{~h}$ in the primary cortical neurons, the CCK-8 assay was applied to evaluate cell viability. (B) After the primary cortical neurons were exposed to $50 \mu M$ of hemin or DMSO for different time points, the CCK-8 assay was applied to evaluate cell viability. (C) Phase-contrast images of cultured cortical neurons exposed to DMSO, 1, 10, 50, and $100 \mu \mathrm{M}$ hemin, respectively. Scale bar, $100 \mu \mathrm{m}$. *, $\mathrm{P}<0.05$ s. Normal (n=5, representative of 5 replicates for each experiment).

essential product in apoptosis (Figure 4A). Direct caspase-3 activity assay confirmed the protective role of PGE1 on caspase-3 (Figure 4B). Moreover, PGE1 appeared to reverse the hemin-induced reduction of anti-apoptotic factor $\mathrm{Bcl}-2$ and thus increased the ratio of $\mathrm{Bcl}-2 / \mathrm{Bax}$ (Figure 4C). Moreover, the anti-apoptotic effect of PGE-1 was confirmed by TUNEL staining (Figure 4D, E). These results indicated that PGE1 could prevent primary cortical neurons from hemin-induced apoptosis.

\section{PGE1 pretreatment induced $\mathrm{Nrf} 2 / \mathrm{HO}-1$ expression in cultured cortical neurons}

To investigate whether the decreased expression of an antioxidant enzyme (HO-1) is positively related to the protection by PGE1, HO-1 and Nrf2 were detected by western blotting (Figure $5 A, B$ ) and immunostaining (Figure 5C). PGE1 pretreatment at the concentrations of 10 or $100 \mathrm{nM}$ significantly increased HO-1 expression 
A
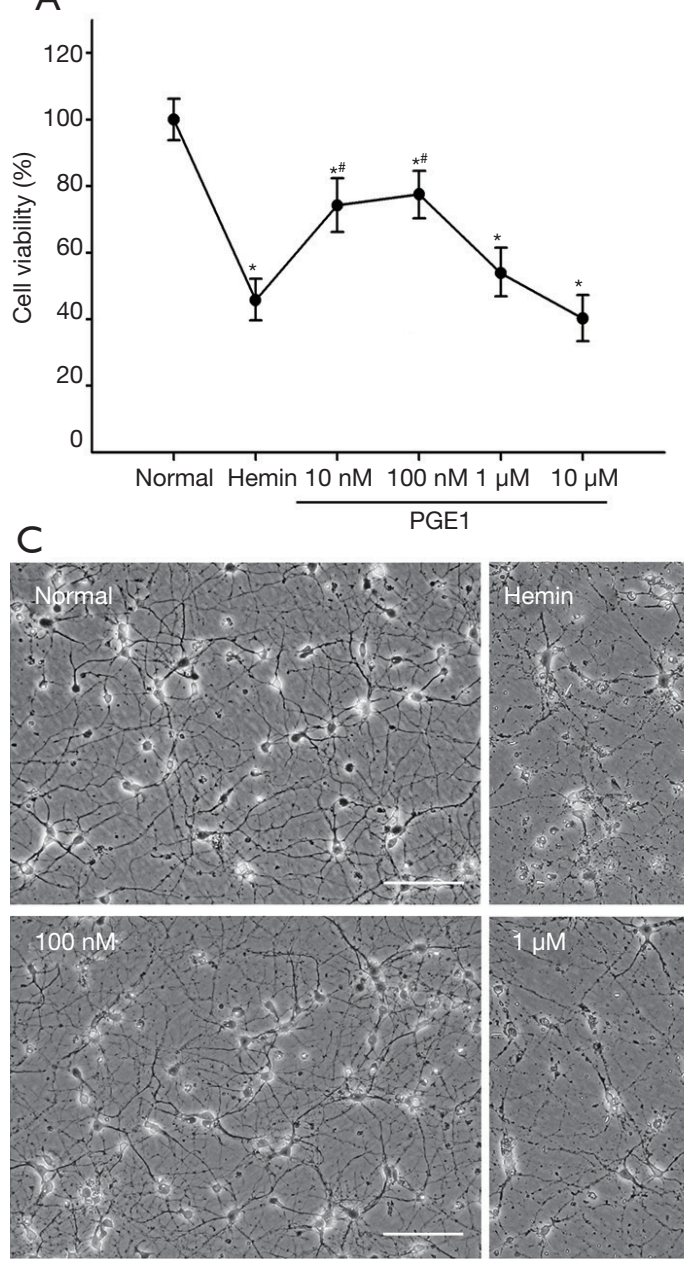

B
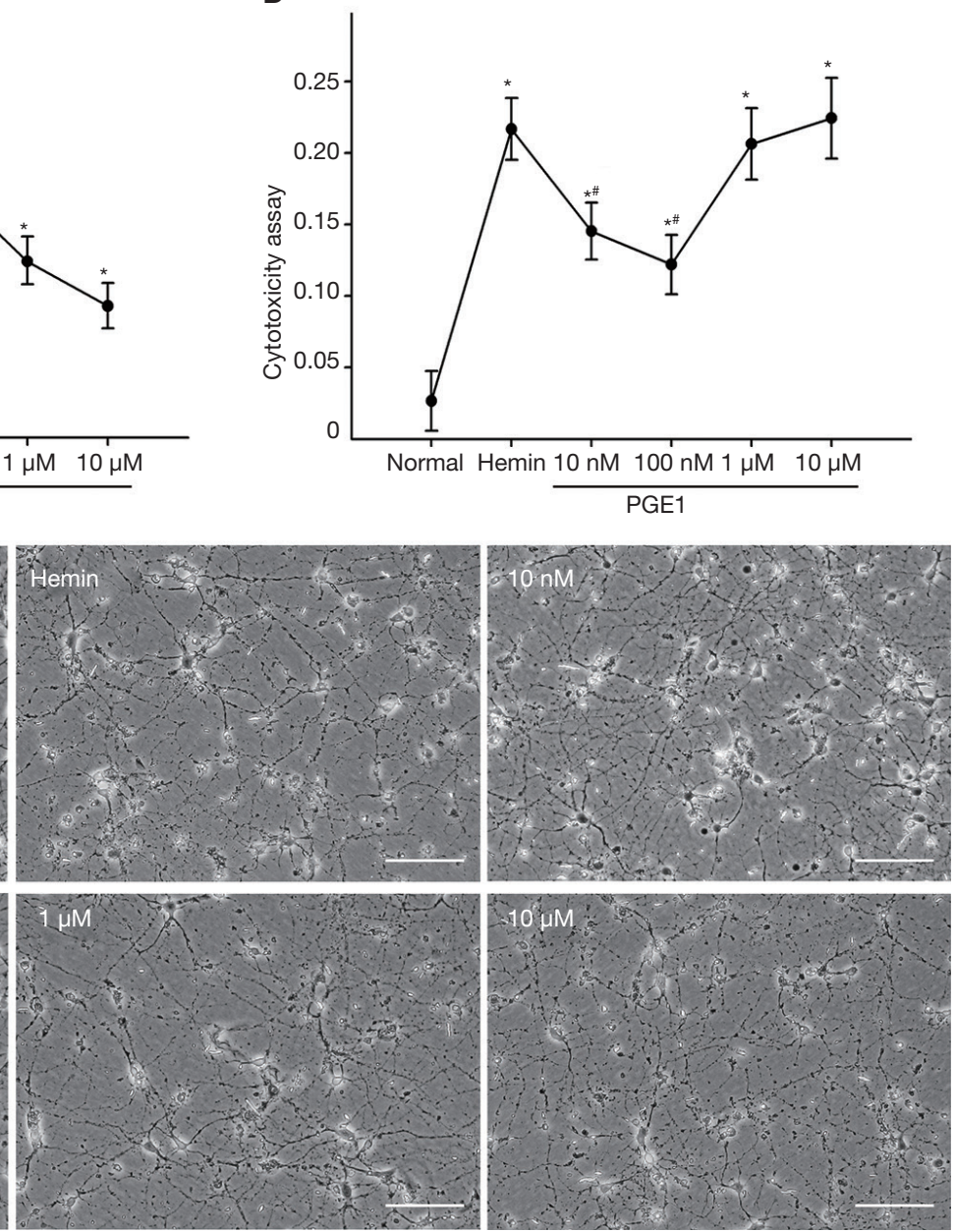

Figure 2 PGE1 pretreatment protected primary cortical neurons against hemin-induced neurotoxicity. (A) The primary cortical neurons were pretreated with or without $10 \mathrm{nM}, 100 \mathrm{nM}, 1 \mu \mathrm{M}$, and $10 \mu \mathrm{M}$ PGE1 for $12 \mathrm{~h}$ and then exposed to $50 \mu \mathrm{M}$ hemin for $24 \mathrm{~h}$. The CCK-8 assay was applied to evaluate cell viability. (B) The neurons were incubated with or without $10 \mathrm{nM}, 100 \mathrm{nM}, 1 \mu \mathrm{M}$, and $10 \mu \mathrm{M}$ of PGE1 for $12 \mathrm{~h}$. Then they were incubated with $50 \mu \mathrm{M}$ hemin for $24 \mathrm{~h}$. The LDH assay was applied to evaluate cell death. (C) The cells' morphological changes for pretreatment with or without $10 \mathrm{nM}, 100 \mathrm{nM}, 1 \mu \mathrm{M}$, and $10 \mu \mathrm{M}$ PGE1 for $12 \mathrm{~h}$, then incubated with $50 \mu \mathrm{M}$ hemin for $24 \mathrm{~h}$. The phase contrast images were captured. Scale bar, $100 \mu \mathrm{m}$. * $\mathrm{P}<0.05$ vs. Normal; ${ }^{*}, \mathrm{P}<0.05$ vs. exposed to hemin ( $\mathrm{n}=5$ ).

(Figure 5 A). Similarly, PGE1 up-regulated the expression of $\mathrm{Nrf} 2$, an endogenous regulator that promotes the transcription of HO-1 (Figure 5B).

\section{Nvf2 knockdown neutralized the protective role of PGE1 on bemin-injured neurons}

To examine whether the protective effect of PGE1 on hemin-injured neurons is Nrf2 dependent, cultured cortical neurons were transfected with si-NC or si-Nrf2. Over $70 \%$ of Nrf2 level was inhibited by si-Nrf2 (Figure $6 A$ ). In these cells, induction of HO-1 expression by PGE1 was dampened (Figure 6B), and the effects of PGE1 on apoptosis were revealed by the levels of cleaved caspase-3 and TUNEL positive cells (Figure 6C, D). Consistently, si$\mathrm{Nrf} 2$ transfection boosted up ROS production compared with si-NC control (Figure 6E). The above results suggested the reducing effects of Nrf2 down-regulation on PGE1 
A
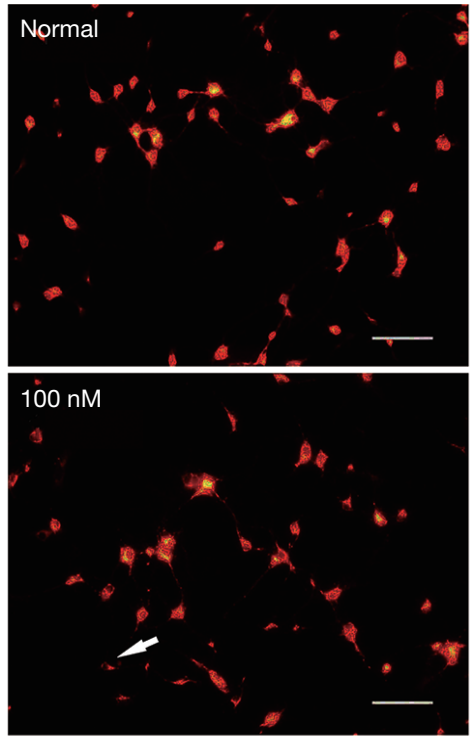

B

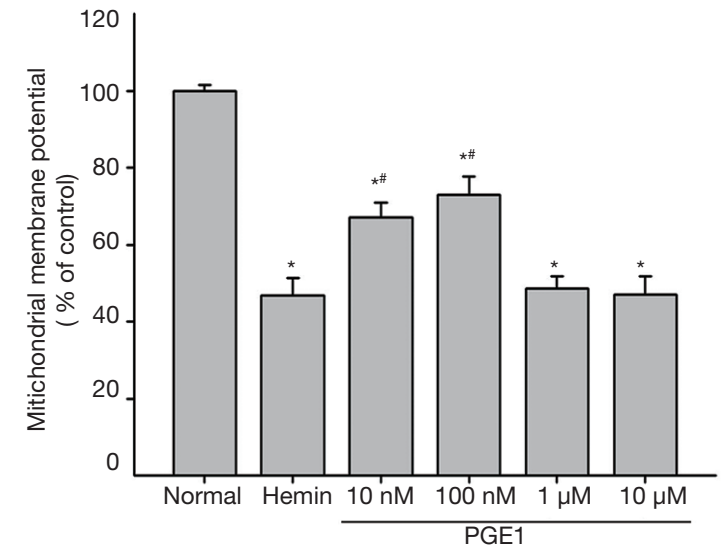

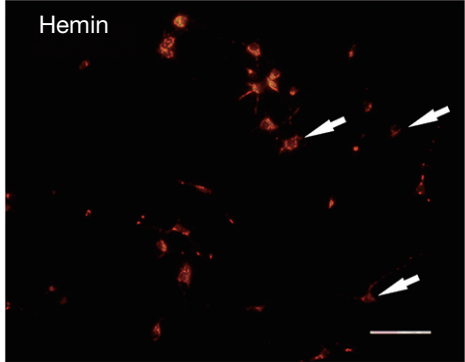
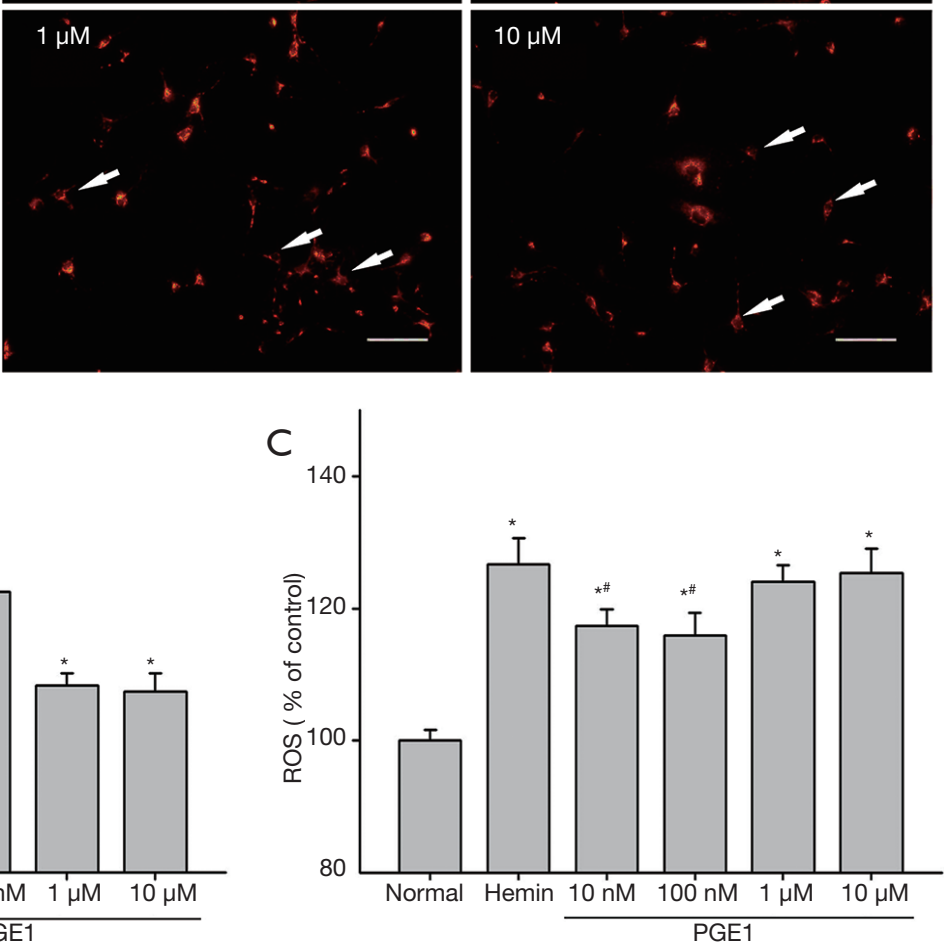

Figure 3 PGE1 improved mitochondrial function in hemin-injured cortical neurons. (A) Representative TMRE staining pictures were analyzed for the measurement of MMP in cultured cortical neurons after different treatments. The arrows indicate the MMP reduction cells. (B) The fluorescent intensity of TMRE in cultured cortical neurons was immediately analyzed under different conditions. (C) The fluorescent intensity of DCFH-DA was analyzed to reflect ROS production after different treatments. Scale bar, $50 \mu \mathrm{m}$. *, $\mathrm{P}<0.05$ vs. Normal; ", $\mathrm{P}<0.05$ vs. exposed to hemin $(\mathrm{n}=5)$.

neuroprotection.

\section{Discussion}

Oxidative stress and mitochondrial dysfunction are regarded as critical factors in hemin-injured neurons' pathogenesis (31). In this study, PGE1 had a protective or drug-induced toxic effect on neurons depending on its concentrations. We have demonstrated that PGE1, at the concentration of 10 or $100 \mathrm{nM}$, significantly protected the hemin-injured neurons, reduced $\mathrm{LDH}$ releasing, cell death, hemin-induced mitochondrial depolarization, and intracellular ROS production, increased Bcl-2/Bax ratio, and decreased neuron apoptosis in vitro ICH model. The protective effect of PGE1 appeared to be inverted U-shaped as higher concentrations than $1 \mu \mathrm{M}$ that was not efficacious in vitro due to the limitation of solubility or cytotoxicity at high concentrations to some extent.

The effect of hematogenous cells and activated neuroglia-derived extravasated blood elements and cytotoxic substances is probably the leading cause of neuronal injury following ICH (32). Hemoglobin of extravasated red blood 
A

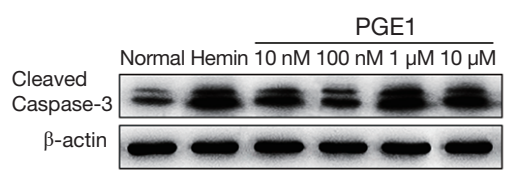

C

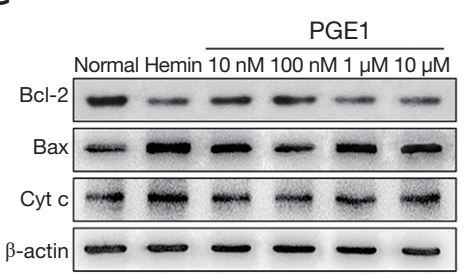

$\mathrm{E}$
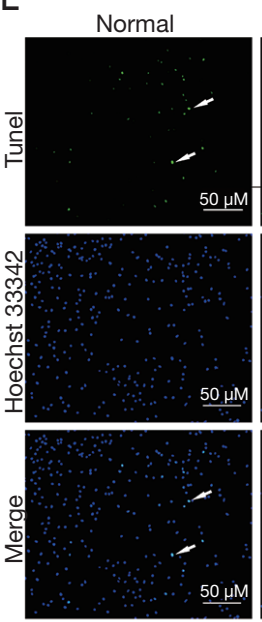

Hemin
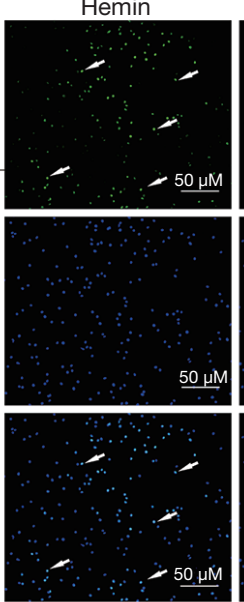

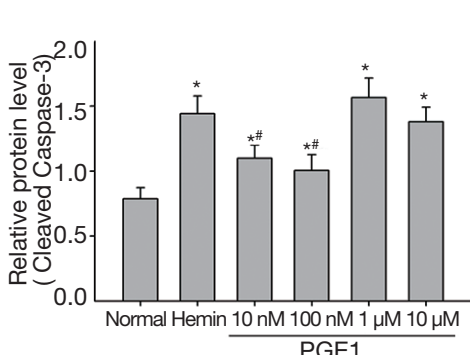

B
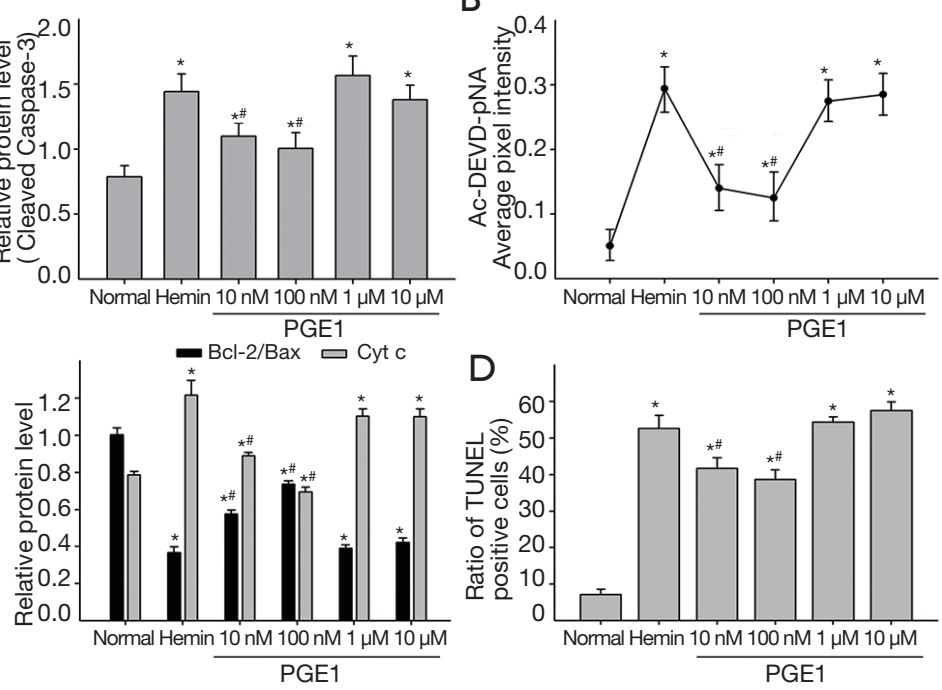

PGE1
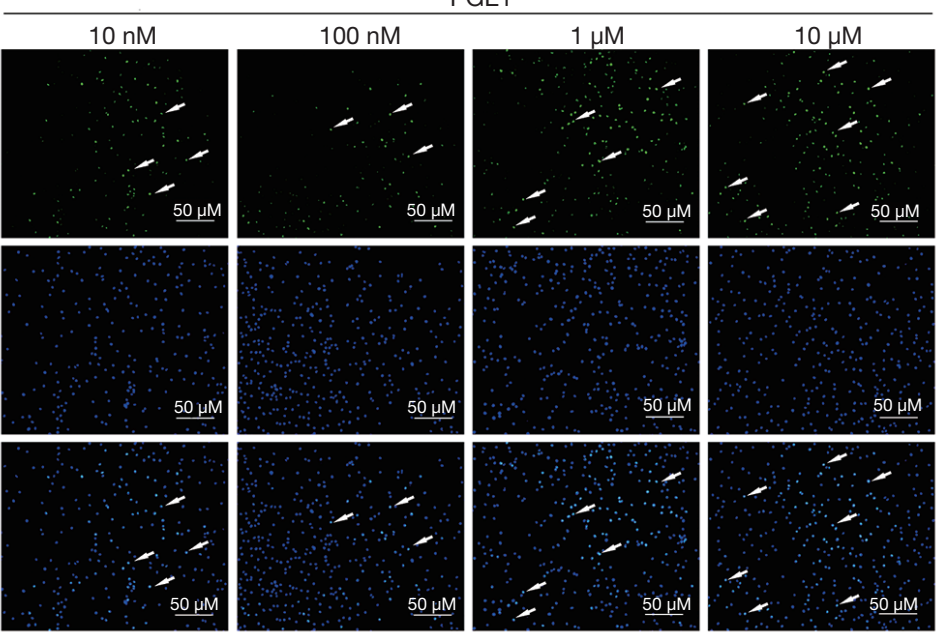

Figure 4 PGE1 rescued primary cortical neurons from hemin-induced apoptosis. (A) The western blotting analysis was carried out to detect the cleaved caspase- 3 expression in cortical neurons pretreated with PGE1 of indicated dose for $12 \mathrm{~h}$, then incubated with hemin for $24 \mathrm{~h}$. The relative expression of the cleaved caspase-3 proteins was normalized to $\beta$-actin, and the ratio of densitometric analysis were plotted into the histogram. (B) Caspase-3 activity was assayed in cortical neurons pretreated with PGE1 of the indicated dose for $12 \mathrm{~h}$, then incubated with hemin for $24 \mathrm{~h}$. (C) Western blotting analysis of Bcl-2, Bax, and Cyt C expressions in cortical neurons pretreated with PGE1 of indicated dose for $12 \mathrm{~h}$, then incubated with hemin for $24 \mathrm{~h}$. The relative expression of the Bcl-2, Bax, and Cyt C were normalized to $\beta$-actin. The results of the ratio for densitometric analysis were plotted into the histogram. (D) TUNEL was performed in cortical neurons pretreated with PGE1 of indicated dose for $12 \mathrm{~h}$, then incubated with hemin for $24 \mathrm{~h}$. TUNEL-positive cells were calculated, and the ratio was normalized to total nuclei. (E) TUNEL staining was designed to detect the apoptotic nuclei(green), and the total number of cells were stained with Hoechst 33342 (blue). The arrows indicate the apoptosis cells. Scale bar, $50 \mu \mathrm{m}$. * $\mathrm{P}<0.05$ vs. Normal; \#, $\mathrm{P}<0.05$ vs. exposed to hemin $(n=5)$.

cells in the brain following ICH degraded into heme and iron, causing harm to the surrounding cells (33). Heme, released by methemoglobin, may rapidly oxidize to form hemin, which activates oxidative damage around brain's hematoma (34,35). PGE1 pretreatment was noted to attenuate oxidative stress, mitochondrial dysfunction, and neuron apoptosis in our work.

Oxidative stress was also reported to exert a prognostic 


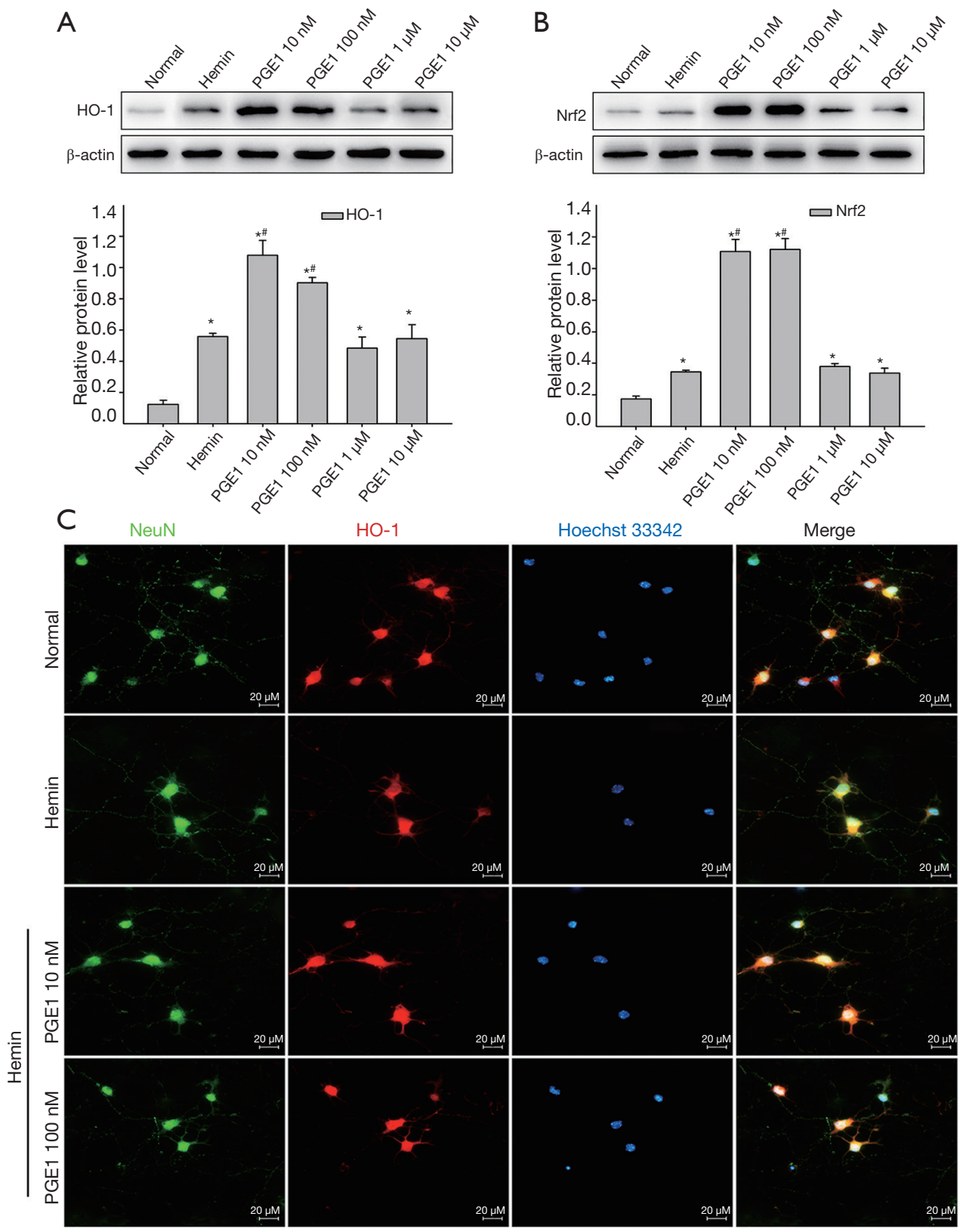

Figure 5 PGE1 pretreatment increased Nrf2/HO-1 expression in cultured cortical neurons. (A) The expression of HO-1 was measured with Western blotting analysis in cortical neurons pretreated with PGE1 for $12 \mathrm{~h}$ and then exposed to hemin for $24 \mathrm{~h}$. The relative expression of HO-1 was normalized to $\beta$-actin, and the ratio of densitometric analysis were plotted into the histogram. (B) Western blotting analysis of Nrf2 expression in cortical neurons pretreated with PGE1 for $12 \mathrm{~h}$ and then exposed to hemin for $24 \mathrm{~h}$. The relative expression of the Nrf2 proteins was normalized to $\beta$-actin. The ratios of densitometric analysis were plotted into the histogram. (C) Immunostaining data showing the expression of NeuN and HO-1 in cortical neurons pretreated with or without 10, $100 \mathrm{nM}$ PGE1 for $12 \mathrm{~h}$, and exposed to $50 \mu \mathrm{M}$ of hemin for $24 \mathrm{~h}$, the nuclei were neutralized with Hoechst 33342, bar $=20 \mu \mathrm{m}$. *, $\mathrm{P}<0.05$ vs. Normal; ${ }^{*}, \mathrm{P}<0.05$ vs. exposed to hemin ( $\mathrm{n}=5$ ). 

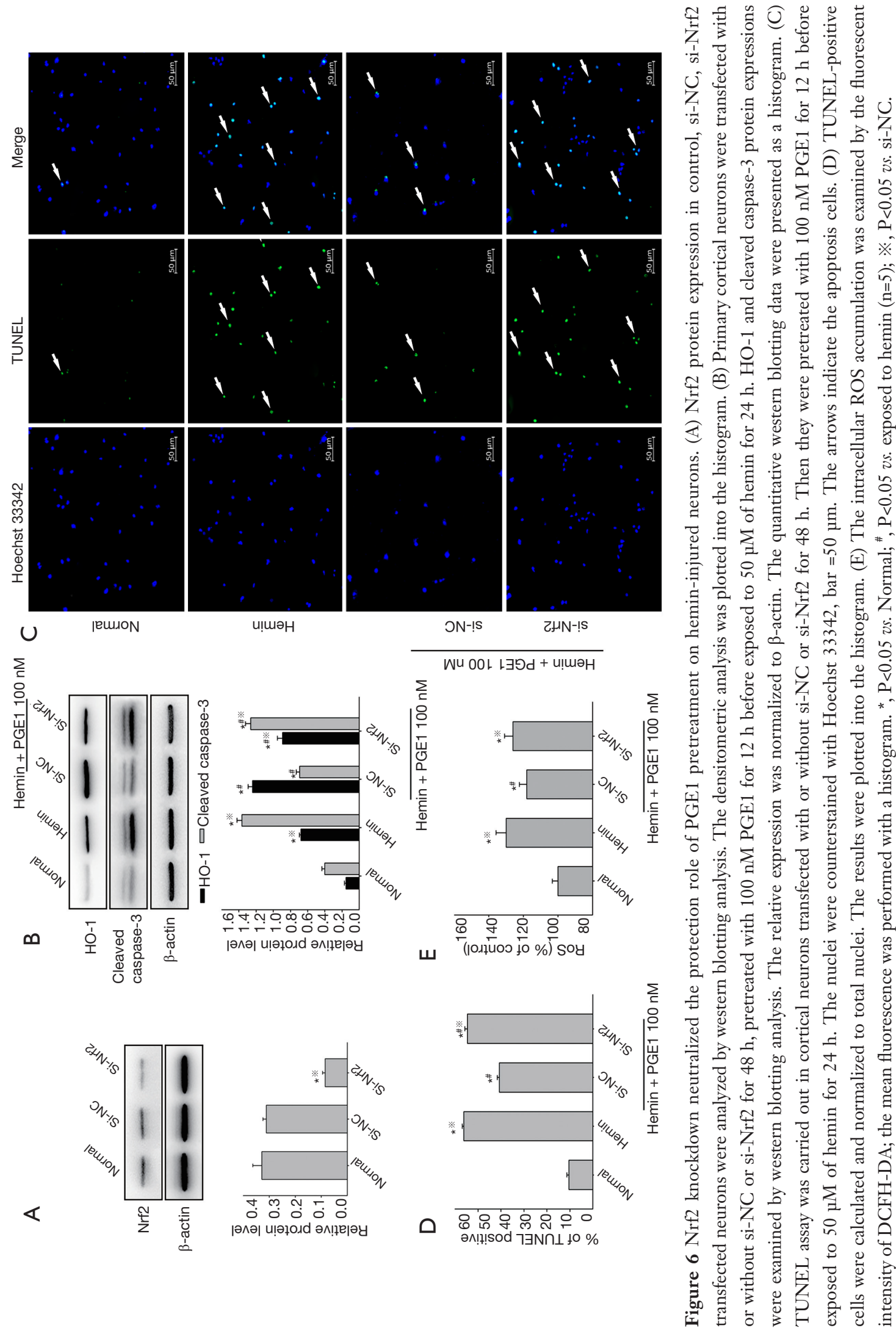
effect in ICH patients $(36,37)$. As a byproduct of cellular metabolism, the oxidase stress ROS is mainly created by the mitochondria in living cells (38). Our data revealed PGE1 might alleviate mitochondrial depolarization and decreased hemin-induced intracellular ROS accumulation at a low concentration (less than $1 \mu \mathrm{M}$ ). Interestingly, higher concentrations of PGE1 (1, or $10 \mu \mathrm{M})$ might not. It indicated that PGE1 potentially impacted the ROS production in hemin-injured cortical neurons. High ROS levels correlate well with apoptosis following ICH by opening mitochondrial permeability transition pore (MPTP) and activating oxidative stress-responsive signaling cascades $(37,39)$. MPTP may also alter the ratio of Bcl-2/Bax, increase the expression of other pro-apoptotic proteins, and trigger Cyt $\mathrm{C}$ release (40). Therefore, the decreased expression of cleaved caspase-3, Bcl-2/Bax, and Cyt C furtherly proved PGE1 (lower concentrations of 10 or $100 \mathrm{nM}$ ) owned the potential roles in anti-apoptosis.

PGE1, brand-named as Alprostadil, has been broadly used to treat clinical diseases. Its pharmacological effects are diverse, as anti-inflammation, membrane stabilization, cytoprotection, inhibition of platelet aggregation to ensure normal perfusion and vasodilation, especially in treating ischemic diseases such as hepatic, myocardial, and cerebral ischemia (41). To explore its application in patients with ICH, we had recruited 40 patients with hypertensive ICH and conducted a clinical trial. We found that PGE1 significantly reduced the volumes of perihematomal and the total hematoma and promoted functional recovery as measured by the Modified Rankin Score (mRS) and National Institutes of Health Stroke Scale (NIHSS) (14). The effect of PGE1 on cytoprotection has been widely reported in preclinical models, but the underlying molecular mechanism needs to be clarified $(42,43)$. Combined with lithium, PGE1 exerted significant roles in a rat cerebral ischemia model (12). Moreover, PGE1 is also reported to prevent neuronal apoptosis in the superficial dorsal horn following chronic constriction of the sciatic nerve in rats (17). In this work, the PGE1 might exert its effect of cytoprotection by reducing oxidative stress and anti-apoptosis.

Our results suggest that PGE1 pretreatment at the concentrations of 10 or $100 \mathrm{nM}$ significantly increased the expression of $\mathrm{HO}-1$ and $\mathrm{Nrf} 2$ that might suppress the cellular oxidative stress and play a neuroprotective effect in vitro. Furthermore, HO-1 exists in most cells to protect them from various stress responses, especially in hemin degradation. HO-1 exerts its antioxidant role in hemin degradation by adapting hemin into cytoprotective molecules such as biliverdin, carbon monoxide, and bilirubin (20). As a transcription factor that regulates antioxidation defense, Nrf2 targets multiple neuroprotective treatments $(44,45)$. In physical condition, $\mathrm{Nrf2}$ is considered as an inactive complex with Keap1 in the cytoplasm. However, after exposure to oxidative stress, Nrf2 can be released from Keap1 repression and translocated to the nucleus where it binds to the antioxidant response element (ARE) and then promotes the transcription of phase II detoxification enzyme genes, such as HO-1 (46). Our data indicated the protective effect of PGE1 on hemin-injured neurons is Nrf2-dependent.

\section{Conclusions}

PGE1 suppresses the oxidative stress of the cells induced by hemin, thereby reducing neuronal apoptosis via intracellular Nrf2/HO-1 signaling.

\section{Acknowledgments}

Funding: This work was financially supported by the National Natural Science Foundation of China (NSFC, No. 81873742, No. 81471188, No. 81671135, No. 81901195), Jiangsu Provincial Key Medical Center and Technology Project of Nantong (MS12017015-1).

\section{Footnote}

Reporting Checklist: The authors have completed the ARRIVE reporting checklist. Available at http://dx.doi. org/10.21037/atm-20-5839

Data Sharing Statement: Available at http://dx.doi. org/10.21037/atm-20-5839

Conflicts of Interest: All authors have completed the ICMJE uniform disclosure form (available at http://dx.doi. org/10.21037/atm-20-5839). The authors have no conflicts of interest to declare.

Ethical Statement: The authors are accountable for all aspects of the research in ensuring that problems related to the accuracy or integrity of any part of the work are adequately investigated and resolved. All animal experiments in this study were approved by the Administration Committee of Experimental Animals [SYXK (Su) 2012-0031] of Jiangsu in compliance with the National Institutes of Health 
Guidelines for the Care and Use of Laboratory Animals.

Open Access Statement: This is an Open Access article distributed in accordance with the Creative Commons Attribution-NonCommercial-NoDerivs 4.0 International License (CC BY-NC-ND 4.0), which permits the noncommercial replication and distribution of the article with the strict proviso that no changes or edits are made and the original work is properly cited (including links to both the formal publication through the relevant DOI and the license). See: https://creativecommons.org/licenses/by-nc-nd/4.0/.

\section{References}

1. $\mathrm{Li} \mathrm{H}, \mathrm{Wu}$ J, Shen H, et al. Autophagy in hemorrhagic stroke: Mechanisms and clinical implications. Prog Neurobiol 2018;163-164:79-97.

2. Tatlisumak T, Cucchiara B, Kuroda S, et al. Nontraumatic intracerebral haemorrhage in young adults. Nat Rev Neurol 2018;14:237-50.

3. van Asch CJ, Luitse MJ, Rinkel GJ, et al. Incidence, case fatality, and functional outcome of intracerebral haemorrhage over time, according to age, sex, and ethnic origin: a systematic review and meta-analysis. Lancet Neurol 2010;9:167-76.

4. Zhu H, Wang Z, Yu J, et al. Role and mechanisms of cytokines in the secondary brain injury after intracerebral hemorrhage. Prog Neurobiol 2019;178:101610.

5. Zhou F, Jiang Z, Yang B, et al. Magnolol exhibits antiinflammatory and neuroprotective effects in a rat model of intracerebral haemorrhage. Brain Behav Immun 2019;77:161-7.

6. Sukumari-Ramesh S, Alleyne CH, Jr., Dhandapani KM. Astrocyte-specific expression of survivin after intracerebral hemorrhage in mice: a possible role in reactive gliosis? J Neurotrauma 2012;29:2798-804.

7. Kreutz RP, Nystrom P, Kreutz Y, et al. Inhibition of platelet aggregation by prostaglandin E1 (PGE1) in diabetic patients during therapy with clopidogrel and aspirin. Platelets 2013;24:145-50.

8. Sheng R, Zhang LS, Han R, et al. Combined prostaglandin $\mathrm{E} 1$ and lithium exert potent neuroprotection in a rat model of cerebral ischemia. Acta Pharmacol Sin 2011;32:303-10.

9. Matsuo H, Shigematsu H. Patient-based outcomes using the Walking Impairment Questionnaire for patients with peripheral arterial occlusive disease treated with LipoPGE1. Circ J 2010;74:365-70.

10. Creutzig A, Lehmacher W, Elze M. Meta-analysis of randomised controlled prostaglandin E1 studies in peripheral arterial occlusive disease stages III and IV. Vasa 2004;33:137-44.

11. Mehrabi MR, Serbecic N, Tamaddon F, et al. Clinical and experimental evidence of prostaglandin E1-induced angiogenesis in the myocardium of patients with ischemic heart disease. Cardiovasc Res 2002;56:214-24.

12. Xie X, Lu W, Chen Y, et al. Prostaglandin E1 Alleviates Cognitive Dysfunction in Chronic Cerebral Hypoperfusion Rats by Improving Hemodynamics. Front Neurosci 2019;13:549.

13. Khalil M, Jux C, Rueblinger L, et al. Acute therapy of newborns with critical congenital heart disease. Transl Pediatr 2019;8:114-26.

14. Cao M, Ke K, Sun H, et al. Effects of prostaglandin E1 on perihematomal tissue after hypertensive intracerebral hemorrhage. Acta Neurol Taiwan 2011;20:172-81.

15. Torres LC, Kulikowski LD, Ramos PL, et al. Disruption of the CREBBP gene and decreased expression of CREB, NFkappaB p65, c-JUN, c-FOS, BCL2 and c-MYC suggest immune dysregulation. Hum Immunol 2013;74:911-5.

16. Shichiri H, Yamamoto K, Tokura M, et al. Prostaglandin E1 reduces the keratinocyte toxicity of sorafenib by maintaining signal transducer and activator of transcription 3 (STAT3) activity and enhancing the cAMP response element binding protein (CREB) activity. Biochem Biophys Res Commun 2017;485:227-33.

17. Kawamura T, Akira T, Watanabe $M$, et al. Prostaglandin E1 prevents apoptotic cell death in superficial dorsal horn of rat spinal cord. Neuropharmacology 1997;36:1023-30.

18. Hamamoto Y, Ogata T, Morino T, et al. Prostaglandin $\mathrm{E} 1$ analog increases spinal cord blood flow at the point of compression during and after experimental spinal cord injury. Spinal Cord 2010;48:149-53.

19. Akaike A, Kaneko S, Tamura Y, et al. Prostaglandin E2 protects cultured cortical neurons against $\mathrm{N}$-methyl-Daspartate receptor-mediated glutamate cytotoxicity. Brain Res 1994;663:237-43.

20. Yang Y, Xi Z, Xue Y, et al. Hemoglobin pretreatment endows rat cortical astrocytes resistance to hemininduced toxicity via Nrf2/HO-1 pathway. Exp Cell Res 2017;361:217-24.

21. Zhang L, Zhang J, Ye Z, et al. Isoflavone ME-344 Disrupts Redox Homeostasis and Mitochondrial Function by Targeting Heme Oxygenase 1. Cancer Res 2019;79:4072-85.

22. Xi G, Strahle J, Hua Y, et al. Progress in translational research on intracerebral hemorrhage: is there an end in 
sight? Prog Neurobiol 2014;115:45-63.

23. Shan H, Li T, Zhang L, et al. Heme oxygenase-1 prevents heart against myocardial infarction by attenuating ischemic injury-induced cardiomyocytes senescence. EBioMedicine 2019;39:59-68.

24. Hedblom A, Hejazi SM, Canesin G, et al. Heme detoxification by heme oxygenase-1 reinstates proliferative and immune balances upon genotoxic tissue injury. Cell Death Dis 2019;10:72.

25. Chen-Roetling J, Kamalapathy P, Cao Y, et al. Astrocyte heme oxygenase-1 reduces mortality and improves outcome after collagenase-induced intracerebral hemorrhage. Neurobiol Dis 2017;102:140-6.

26. Shah SA, Khan M, Jo MH, et al. Melatonin Stimulates the SIRT1/Nrf2 Signaling Pathway Counteracting Lipopolysaccharide (LPS)-Induced Oxidative Stress to Rescue Postnatal Rat Brain. CNS Neurosci Ther 2017;23:33-44.

27. Obuchowicz E, Kowalski J, Labuzek K, et al. Amitriptyline and nortriptyline inhibit interleukin-1 release by rat mixed glial and microglial cell cultures. Int J Neuropsychopharmacol 2006;9:27-35.

28. Cao M, Tan X, Jin W, et al. Upregulation of Ras homolog enriched in the brain (Rheb) in lipopolysaccharide-induced neuroinflammation. Neurochem Int 2013;62:406-17.

29. Damulewicz M, Loboda A, Jozkowicz A, et al. Interactions Between the Circadian Clock and Heme Oxygenase in the Retina of Drosophila melanogaster. Molecular Neurobiology 2017;54:4953-62.

30. Zhang Q, Chen S, Yu S, et al. Neuroprotective effects of pyrroloquinoline quinone against rotenone injury in primary cultured midbrain neurons and in a rat model of Parkinson's disease. Neuropharmacology 2016;108:238-51.

31. Yang F, Shan Y, Tang Z, et al. The Neuroprotective Effect of Hemin and the Related Mechanism in Sevoflurane Exposed Neonatal Rats. Front Neurosci 2019;13:537.

32. Zhao X, Grotta J, Gonzales N, et al. Hematoma resolution as a therapeutic target: the role of microglia/macrophages. Stroke 2009;40:S92-4.

33. Leclerc JL, Li C, Jean S, et al. Temporal and agedependent effects of haptoglobin deletion on intracerebral hemorrhage-induced brain damage and neurobehavioral outcomes. Exp Neurol 2019;317:22-33.

34. Aronowski J, Zhao X. Molecular pathophysiology of cerebral hemorrhage: secondary brain injury. Stroke 2011;42:1781-6.

35. Felberg RA, Grotta JC, Shirzadi AL, et al. Cell death in experimental intracerebral hemorrhage: the "black hole" model of hemorrhagic damage. Ann Neurol 2002; 51:517-24.

36. Xia M, Chen W, Wang J, et al. TRPA1 ActivationInduced Myelin Degradation Plays a Key Role in Motor Dysfunction After Intracerebral Hemorrhage. Front Mol Neurosci 2019;12:98.

37. Hu YL, Wang H, Huang Q, et al. MicroRNA-23a$3 p$ promotes the perihematomal edema formation after intracerebral hemorrhage via ZO-1. Eur Rev Med Pharmacol Sci 2018;22:2809-16.

38. Hantson P. Mechanisms of toxic cardiomyopathy. Clin Toxicol (Phila) 2019;57:1-9.

39. Eleftheriadis T, Pissas G, Liakopoulos V, et al. Cytochrome $\mathrm{c}$ as a Potentially Clinical Useful Marker of Mitochondrial and Cellular Damage. Front Immunol 2016;7:279.

40. Gao GZ, Wu C, Zhai KF, et al. The Protective Effect of Sulfated Pachymaran on 1-Methyl-4-Phenyl-1,2,3,6Tetrahydropyridine-Induced Neuronal Apoptosis in Mice. Current Topics In Nutraceutical Research 2019;17:64-8.

41. Han R, Gao B, Sheng R, et al. Synergistic effects of prostaglandin $\mathrm{E} 1$ and lithium in a rat model of cerebral ischemia. Acta Pharmacol Sin 2008;29:1141-9.

42. Li F, He B, Ma X, et al. Prostaglandin E1 and Its Analog Misoprostol Inhibit Human CML Stem Cell Self-Renewal via EP4 Receptor Activation and Repression of AP-1. Cell Stem Cell 2017;21:359-73 e5.

43. $\mathrm{Wu} \mathrm{H}, \mathrm{Wu} \mathrm{T}$, Hua $\mathrm{W}$, et al. PGE2 receptor agonist misoprostol protects brain against intracerebral hemorrhage in mice. Neurobiol Aging 2015;36:1439-50.

44. Wei CC, Kong YY, Li GQ, et al. Nicotinamide mononucleotide attenuates brain injury after intracerebral hemorrhage by activating $\mathrm{Nrf} 2 / \mathrm{HO}-1$ signaling pathway. Sci Rep 2017;7:717.

45. Zhang L, Wang H, Fan Y, et al. Fucoxanthin provides neuroprotection in models of traumatic brain injury via the Nrf2-ARE and Nrf2-autophagy pathways. Sci Rep 2017;7:46763.

46. Choi EM, Suh KS, Kim YJ, et al. Glabridin Alleviates the Toxic Effects of Methylglyoxal on Osteoblastic MC3T3-E1 Cells by Increasing Expression of the Glyoxalase System and Nrf2/HO-1 Signaling and Protecting Mitochondrial Function. J Agric Food Chem 2016;64:226-35.

Cite this article as: Shen J, Cao MS, Zhou T, Chen Y, Liang J, Song Y, Xue C, Cao MH, Ke K. PGE1 triggers Nrf2/HO-1 signal pathway to resist hemin-induced toxicity in mouse cortical neurons. Ann Transl Med 2021;9(8):634. doi: 10.21037/atm-20-5839 